\title{
Guiding of an electromagnetic pulse in a plasma immersed in combined wiggler and axial magnetic fields
}

\author{
Min Sup Hur \\ Korea Electrotechnology Research Institute, Changwon, Kyongnam 641-120, South Korea \\ Jonathan S. Wurtele \\ University of California, Berkeley and Lawrence Berkeley National Laboratory, \\ Berkeley, CA 94720, USA \\ Gregory Penn \\ Lawrence Berkeley National Laboratory, Berkeley, CA 94720, USA
}

LBNL Technical Report, June 2008

Published as Physics Letters A 372 (2008) 3452-3455.

\section{Disclaimer}

This document was prepared as an account of work sponsored by the United States Government. While this document is believed to contain correct information, neither the United States Government nor any agency thereof, nor The Regents of the University of California, nor any of their employees, makes any warranty, express or implied, or assumes any legal responsibility for the accuracy, completeness, or usefulness of any information, apparatus, product, or process disclosed, or represents that its use would not infringe privately owned rights. Reference herein to any specific commercial product, process, or service by its trade name, trademark, manufacturer, or otherwise, does not necessarily constitute or imply its endorsement, recommendation, or favoring by the United States Government or any agency thereof, or The Regents of the University of California. The views and opinions of authors expressed herein do not necessarily state or reflect those of the United States Government or any agency thereof, or The Regents of the University of California.

Ernest Orlando Lawrence Berkeley National Laboratory is an equal opportunity employer.

This work was supported by the Director, Office of Science,

High Energy Physics, U.S. Department of Energy under contract number DE-AC02-05CH11231. 


\title{
Guiding of an electromagnetic pulse in a plasma immersed in combined wiggler and axial magnetic fields
}

\author{
Min Sup Hur* \\ Korea Electrotechnology Research Institute, Changwon, Kyongnam 641-120, South Korea \\ Jonathan S. Wurtele \\ University of California, Berkeley and Lawrence Berkeley National Laboratory, Berkeley, CA 94720, USA \\ Gregory Penn \\ Lawrence Berkeley National Laboratory, Berkeley, CA 94720, USA
}

(Dated: 11 December 2007)

\begin{abstract}
We present a new plasma-based method of guiding an electromagnetic pulse. The scheme consists of an inhomogeneous magnetic field and a uniform density plasma, in contrast to existing schemes that rely on transverse plasma density gradients but need not be magnetized. The refractive index of a magnetized plasma depends on the strength and direction of the magnetic field as well as the plasma density. A guiding channel is formed by using field inhomogeneity to generate the desired transverse profile of the index of refraction. The concept is analyzed with an envelope equation and, for the specific example of a wiggler magnetic field, with a two-dimension particle-in-cell simulation. A simplified model of this scheme as producing a magnetic wall in analogy to metallic waveguides is presented, for which corresponding approximate relations for the guided mode axial wavelength and radius are derived as functions of the plasma and magnetic field parameter. These are seen to be in good agreement with particle-in-cell simulation results. Since the desired inhomogeneity of the refractive index can be made easily when the electromagnetic wave frequency is close to the cyclotron frequency, this guiding scheme is most readily applied in the microwave regime.
\end{abstract}

PACS numbers: 52.25.Xz; 52.35.Hr; 52.38.Hb

Keywords: Wave guiding; Magnetized plasma; Particle-in-cell simulation

It is important in many applications, such as laserdriven plasma-based accelerators $[1,2]$, to propagate intense electromagnetic pulses over distances much larger than the diffraction length. The propagation distance in vacuum is characterized by the Rayleigh length $Z_{R}=$ $\pi r_{s}^{2} / \lambda$, where $r_{s}$ and $\lambda$ are the wave spot size and wavelength, respectively. The high-intensity pulses are guided using a plasma channel, where the plasma density is low at the pulse propagation axis and increases in the radial direction. Such a density structure produces an index of refraction decreasing with radial position [3-6]. The density gradient is obtained either with a capillary discharge or with careful timing with a gas jet that is thermally expanding in the radial direction. These plasma structures handle intense fluxes, their optical properties readily varied by changing the delay between, for example, the application of a heating pulse to the center of the plasma and time at which the high intensity pulse enters the channel. In contrast, high power microwaves are guided by metallic structures. We propose here a plasma-based guide for microwaves that requires only a gradient in the external magnetic field and a homogenous plasma density. The advantage is that the magnetic field is readily varied and known to high precision. Thus, the characteristics of the guiding are easily modified by adjusting the

${ }^{*}$ Tel.: +82-55-280-1462; fax: +82-55-280-1469; email: benjhur@ gmail.com field.

Then the basic idea is varying the refractive index by an external non-uniform magnetic field in a uniform plasma. The refractive index of a magnetized plasma is a function of the magnetic field for a given frequency and polarization of the EM wave. Consider a right-handcircular polarized wave (R-wave), whose refractive index in a uniformly magnetized plasma is

$$
\eta=\sqrt{1-\frac{\omega_{p}^{2}}{\omega^{2}} \frac{\omega}{\omega-\Omega}},
$$

where $\omega_{p}=\sqrt{e^{2} n_{0} / \varepsilon_{0} m}$ is the plasma frequency, $-e$ is the electron charge, $m$ is the electron mass, $n_{0}$ the plasma density, $\varepsilon_{0}$ the permitivity of free space, $\omega$ the electrmagnetic wave frequency, and $\Omega=e B / m \gamma$ the cyclotron frequency, $B$ is the magnetic field strength and $\gamma=E / m c^{2}$ the relavistic factor, where $E$ is the electron energy.

A simple illustration of the idea is given by assuming an axial field with a radial variation. As the magnetic field increases radially, the refractive index decreases, and becomes zero at the cutoff. This behavior yields the required negative radial gradient of the refractive index. Because the maximum value of the laboratory magnetic fields is of order a few Tesla, the magnetic guiding is most efficient in the microwave regime. Hence, all the simulations presented in this paper specified frequencies in the microwave range.

To easily realize a transversely increasing magnetic field in the laboratory, it should satisfy $\nabla \times \vec{B}=0$, i.e., 
it should be an external field, rather than one induced by a current inside the plasma. That can be achieved by a set of alternating magnets, which is the same as a normal magnetic wiggler except that the polarization of magnets in each pair is anti-parallel, producing an oscillating longitudinal field on axis. In the slab geometry, the alternating field can be represented by

$$
\begin{aligned}
& B_{z}=B_{0}+B_{w} \sin \left(k_{w} z\right) \cosh \left(k_{w} y\right) \\
& B_{y}=-B_{w} \cos \left(k_{w} z\right) \sinh \left(k_{w} y\right), \quad B_{x}=0
\end{aligned}
$$

where $B_{0}$ is a constant axial magnetic field, $B_{w}$ the wiggler amplitude, and $k_{w}$ the wiggler wavenumber $\left(k_{w}=\right.$ $\left.2 \pi / \lambda_{w}\right)$. In the cylindrical geometry, $\cosh \left(k_{w} y\right)$ and $\sinh \left(k_{w} y\right)$ would be replaced by the modified Bessel functions $I_{0}\left(k_{w} y\right)$ and $I_{1}\left(k_{w} y\right)$. The two dimensional particlein-cell (PIC) code XOOPIC [7] is used to study the guiding properties of such a system, as shown in Fig. 1. The frequency of the EM wave and the plasma frequency are $\omega=1.88 \times 10^{11} \mathrm{~s}^{-1}$ and $\omega_{p}=0.2 \omega$, respectively; the wiggler parameters are $B_{w}=0.1 \mathrm{~T}, B_{0}=0.5 \mathrm{~T}$, and the wiggler wavelength $\lambda_{w}=0.1 \mathrm{~m}$. The wave was launched at the left boundary with the focal point $0.1 \mathrm{~m}$ away from the launching position. The propagation distance of the wave is about $1 \mathrm{~m}$. An initially Gaussian transverse profile $\exp \left(-y^{2} / y_{s}^{2}\right)$ is used with the spot size $y_{s}$ at the focus was set at $0.02 \mathrm{~m}$. The two-dimensional images of the unguided $\left(B_{w}=0\right)$ and guided $\left(B_{w}=0.1 \mathrm{~T}\right)$ cases are denoted as (a) and (b) in Fig. 1. The intensity of the guided wave is preserved over many diffraction lengths in the guided case, while the unguided pulse rapidly diffracts. The guiding by the wiggler field is more evident in Fig. 1 (c), where the peak intensity of the wave at the propagation axis is plotted. The intensity of the unguided wave monotonically decreases by the diffraction, while the guided wave intensity oscillates. We interpret the oscillation as indicating a mismatch between the focal properties of the channel and the initial spot size.

To find a better matched case, the channel size in the slab geometry is estimated starting from the twodimensional envelope equation:

$$
\frac{\partial \hat{E}}{\partial z}=\frac{i}{2 k} \frac{\partial^{2} \hat{E}}{\partial y^{2}}-\frac{i k}{2}\left(1-\eta^{2}\right) \hat{E}
$$

where $\hat{E}$ is the complex slowly varying amplitude of electric field, and $\eta$ the refractive index. The first term in the right-hand-side is the diffraction term. The wave profile can be characterized by

$$
\hat{E}=\hat{E}_{0} R(z) \exp [i \delta(z)] \exp \left[-\frac{y^{2}}{y_{s}(z)^{2}}[1+i \alpha(z)]\right],
$$

where $\alpha, R$, and $\delta$ are real-valued function of $z$, and $y_{s}$ the spot size of the wave. Inserting Eq. (4) into Eq. (3) and taking the real and imaginary parts yields

$$
\frac{R^{\prime}}{R}+\frac{2 y^{2}}{y_{s}^{2}} \frac{y_{s}^{\prime}}{y_{s}}=\frac{\alpha}{k y_{s}^{2}}\left(1-\frac{4 y^{2}}{y_{s}^{2}}\right)
$$
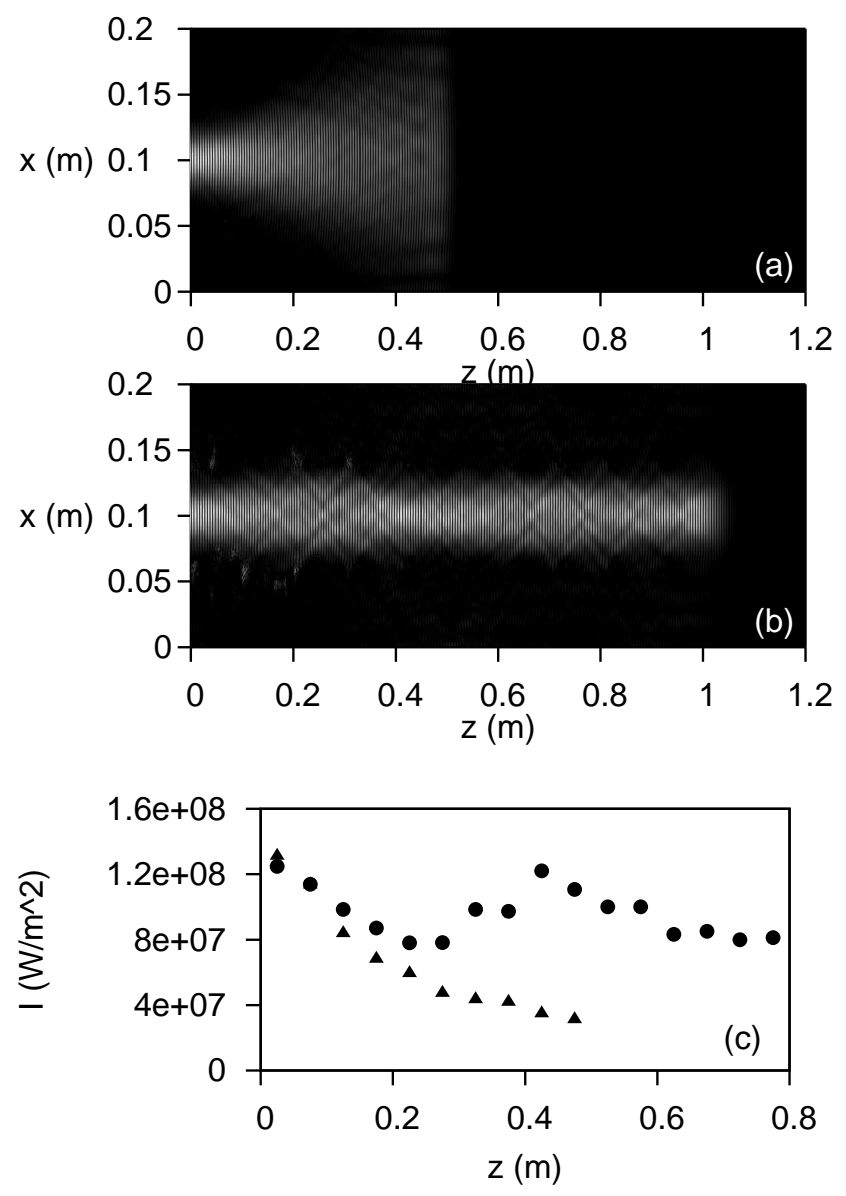

FIG. 1: Wave intensity profiles for (a) unguided and (b) periodically guided cases. (c) Peak intensity of the wave at the propagation axis, comparing unguided (triangles) and guided (circles) geometries.

and

$$
\begin{aligned}
\delta^{\prime}+ & \alpha \frac{2 y^{2}}{y_{s}^{2}} \frac{y_{s}^{\prime}}{y_{s}}-\frac{y^{2}}{y_{s}^{2}} \alpha^{\prime} \\
& =-\frac{1}{k y_{s}^{2}}+\frac{2 y^{2}}{k y_{s}^{2}}\left(1-\alpha^{2}\right)-\frac{k}{2}\left(1-\eta^{2}\right),
\end{aligned}
$$

where the prime represents the derivative in terms of $z$. In the region where the channel is narrowest, i.e., $\sin \left(k_{w} z\right) \simeq 1$, the cyclotron frequency $\Omega$ for the wiggler field Eq. (2) is defined by

$$
\Omega \simeq \Omega_{0}+\Omega_{w} \cosh k_{w} y
$$

where $\Omega_{0}=e B_{0} / m$ and $\Omega_{w}=e B_{w} / m$, respectively. Then the R-wave dispersion Eq. (1) can be expanded up to the second order in $y$ as

$$
1-\eta^{2} \simeq \frac{\omega_{p}^{2}}{\omega^{2}}\left[1+\frac{\Omega_{s}}{\omega}+\frac{\Omega_{s}^{2}}{\omega^{2}}+\frac{\Omega_{w}}{2 \omega}\left(1+\frac{2 \Omega_{s}}{\omega}\right) k_{w}^{2} y^{2}\right],
$$

where $\Omega_{s} \equiv \Omega_{0}+\Omega_{w}$. The $y^{2}$ terms in Eq. (5) yield $\alpha=-y_{s}^{\prime} y_{s} / 2$. Substituting this expression and Eq. (8) 
into Eq. (6) and isolating the $y^{2}$ terms of the result yields the equation of the spot size $y_{s}$ :

$$
y_{s} \frac{\partial^{2} y_{s}}{\partial z^{2}}-\frac{4}{k^{2} y_{s}^{2}}+\frac{k_{w}^{2} \omega_{p}^{2} \Omega_{w}}{2 \omega^{3}}\left(1+\frac{2 \Omega_{s}}{\omega}\right) y_{s}^{2}=0 .
$$

Since the channel size is a function of the pulse position $z$, there is no global equilibrium of the spot size. Instead the local equilibrium of $y_{s}$ can be calculated at the narrowest region of the channel as

$$
y_{s \mathrm{eq}}^{2}=\frac{2}{k k_{w}} \frac{\omega}{\omega_{p}} \sqrt{\frac{2 m \omega}{e B_{w}}\left(1+\frac{2 \Omega_{s}}{\omega}\right)^{-1}} .
$$

For the parameters used in Fig. 1, $y_{s \text { eq }}=0.0284 \mathrm{~m}$, for which the FWHM (full-width-half-maximum) of the wave intensity is $0.0334 \mathrm{~m}$. In Fig. 2, the FWHM of the EM wave was measured from the same simulation as in Fig. 1. The spatially averaged FWHM is 0.032 , which is very close to the theoretical value.

We describe approximate relations for the radius of the magneto-plasma channel and the guided mode axial wavelength. From Eq. (1), the stop-band of the R-wave is calculated as $\omega\left(1-\omega_{p}^{2} / \omega^{2}\right)<\Omega<w$, where the lower limit is the cutoff and the upper limit the resonance. The behavior of the refractive index near those two points is approximated as follows. The gradient of the refractive index is, from Eq. (1),

$$
\frac{\mathrm{d} \eta}{\mathrm{d} \Omega}=-\frac{\omega_{p}^{2}}{2 \eta \omega(\omega-\Omega)^{2}} .
$$

It is seen from Eq. (11) that the gradient of $\eta$ is very large at the cutoff $(\eta=0)$ and the resonance $(\omega=\Omega)$, which implies that $\eta$ changes very rapidly near those two points. Hence, for a smoothly varying spot over the channel, the refractive index near the cutoff can be approximated by a step function. The second approximation is that the R-mode wave field is completely zero in the stop-band. These two assumptions make the cutoff line look similar to the metallic wall of the waveguide, i.e., a magnetic wall. The derivation of the dispersion relation for this situation is almost the same as in the conventional waveguide except the boundary conditions. The longitudinal field in the magnetic channel does not have a definite boundary condition, since the electric field oscillating parallel to the magnetic field is not influenced by the magnetic field. On the other hand, the transverse field should be zero at the boundary, since the stop-band prohibits the propagation of the transversely oscillating field. If we apply this idea to the wigglerplasma system, then the waveguide structure looks like curved walls repeating periodically. In this paper, for the convenience in analysis, we consider the case where the magnetic wall is straight along the wave propagation direction and varying transversely only. The typical procedure to find the dispersion in the metallic waveguide is to determine first the longitudinal field and the transverse fields are derived from it. In the magnetic wall

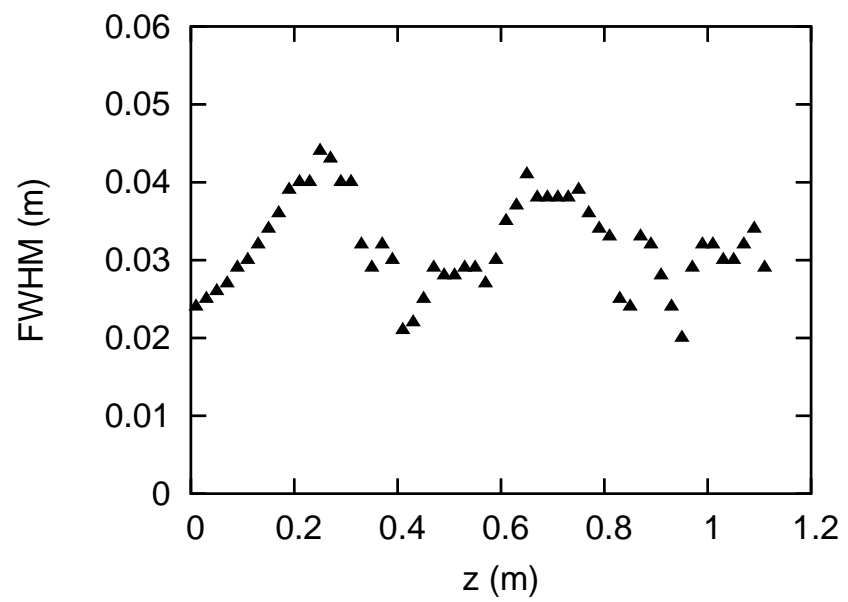

FIG. 2: Evolution of the pulse FWHM. The data marked by triangles are for the same guided simulation parameters as used in Fig. 1. The initial spot size was $r_{0}=2.0 \mathrm{~cm}$.

system, the procedure is reversed, since the boundary condition is different. The transverse field (R-wave) is found first with the zero-field boundary condition at the magnetic wall (the cutoff), and the longitudinal field is derived from it. We consider the system which is homogeneous in $z$-direction and bounded by the magnetic wall in $y$-direction. The wave propagates in $z$-direction. The electric field has the form $\vec{E}=\vec{E}_{0}(y) \exp (i k z-i \omega t)$, where $\vec{E}_{0}(y)=\left[E_{x}(y), E_{y}(y), E_{z}(y)\right]$. From the wave equation, $E_{x}$ satisfies

$$
\partial^{2} E_{x} / \partial y^{2}+\gamma^{2} E_{x}=0, \quad \gamma^{2}=\tilde{\epsilon} \omega^{2} / c^{2}-k^{2},
$$

where $\tilde{\epsilon}$ is the relative dielectric constant of the plasma defined by $\tilde{\epsilon} \equiv \epsilon / \epsilon_{0}=1-\omega_{p}^{2} / \omega^{2}$. The solution of Eq. (12) is a superposition of various sinusoidal modes with the boundary condition $E_{x}=E_{x}=0$ at the cutoff positions $y=0$ and $y=L$. These boundary conditions are satisfied only when $\gamma=n \pi / L$ with $n$ an integer. Hence, the dispersion relation takes the following form:

$$
\lambda=2 \pi / \sqrt{\left(1-\frac{\omega_{p}^{2}}{\omega^{2}}\right) \frac{\omega^{2}}{c^{2}}-\pi^{2} / L^{2}} .
$$

Here $L$, the distance between the two cutoff's in the magneto-plasma channel, is a function of the axial and wiggler magnetic field. In the region where the channel is narrowest $\left(\sin k_{w} z=1\right)$,

$$
L=\frac{2}{k_{w}} \operatorname{arccosh}\left[\frac{B_{c}-B_{0}}{B_{w}}\right],
$$

where $B_{c}$ is the magnetic field at the cutoff, derived from the condition of $\eta=0$ in Eq. (1). Equation (13) with $L$ given by Eq. (14) was compared to the simulation results in Fig. 3. We plotted the wavelength of the EM wave as a function of the axial magnetic field $B_{0}$ for a given wave frequency. The simulation results agree reasonably well with the theory. 


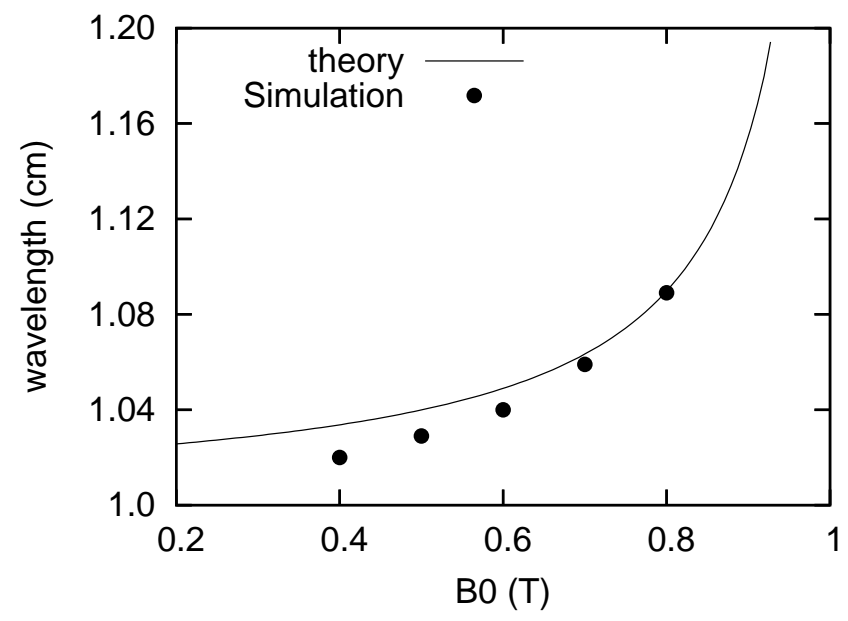

FIG. 3: The wavelength $(\lambda)$ as a function of the axial magnetic field $B_{0}$. The wave parameters are $\lambda=0.01 \mathrm{~m}, r_{s}=2 \lambda$ and $\omega_{p}=0.2 \omega$. The theory curve is from Eq. (13).

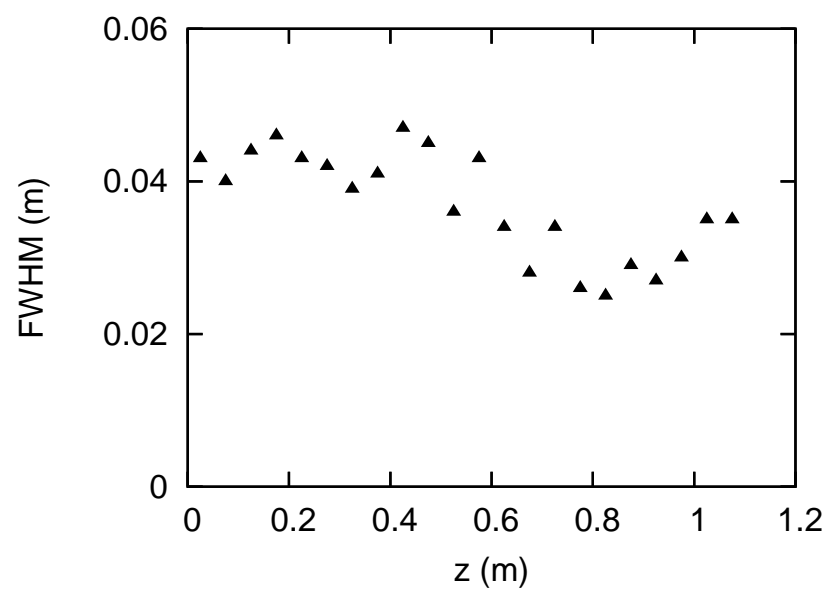

FIG. 4: Guiding of an intense electromagnetic wave with a normalized vector potential $a=0.3$ and an initial spot $r_{0}=$ $3.5 \mathrm{~cm}$. In this case, a smaller axial field $\left(B_{0}=0.2 \mathrm{~T}\right)$ was used. Wiggler field and all other parameters are the same as in Fig. 1.

The magnetic guiding scheme is well-suited to the transmission of very high power microwaves, since the plasma-wiggler system is not subject to the vacuum breakdown as in the conventional waveguide. The normalized amplitude of the wave in Fig. 1 is $a=0.001$ corresponding to $I=2.7 \times 10^{8} \mathrm{~W} / \mathrm{m}^{2}$ or $E=0.32 \mathrm{MV} / \mathrm{m}$, which is mild enough to be guided also in a metallic waveguide. We have checked that similar guiding can be obtained even for relativistic wave intensities. Fig. 4 presents the FWHM of the EM wave for $a=0.3$, with the initial spot $r_{0}=3.5 \mathrm{~cm}$. A smaller axial field $B_{0}=0.2 \mathrm{~T}$ was used. All the other parameters are the same as those used in Fig. 1. In this case the wave is being guided by the magnetic channel. The field strength for $a=0.3$ is about $100 \mathrm{MV} / \mathrm{m}$, which is almost the vacuum break- down limit. The high power microwave transfer suggests the possibility of the system being used as a compact accelerator.

Another application of the magneto-plasma channel is the tunable waveguide. The magnetic channel width, which can be roughly defined as the distance between the transverse position where the wave is at cutoff, is very robust to plasma density fluctuation because, in the regime where $\omega \sim \Omega_{0} \gg \omega_{p}$, the cutoff of the Rmode $\left(0.5 \Omega\left[1+\sqrt{1+4 \omega_{p}^{2} / \Omega_{0}^{2}}\right]\right)$ depends only weakly on the plasma frequency. This robustness endows the system with good controllability: the focusing, guiding, and bending of the EM beam are adjusted by varying the external wiggler field strength.

In summary, we proposed and studied a new concept for wave guiding in a magnetized plasma. A nonuniform magnetic field transversely increasing from the wave propagation axis creates a refractive index with a negative transverse (radial) gradient, as required for guiding. A specific example of the idea, using a wiggler magnetic field, was simulated with two-dimensional particle-in-cell simulations. A simple model in which the stop-band of the R-wave is regarded as a magnetic wall which guides the wave leads to an approximate relation between the wavelength and the channel radius. The relation showed good agreement with two-dimensional simulations. The simulations showed that the magnetic channel is effective even for the guiding of a relativistic wave. For a more formal analysis of the guiding in the magneto-plasma channel, an analysis based on ray tracing methods will be performed.

\section{Acknowledgments}

We thank Prof. Lazar Friedland for very useful and constructive discussion. This work was supported by the Director, Office of Science, High Energy Physics, U.S. Department of Energy under Contract No. DEAC02-05CH11231.

\section{References}

[1] T. Tajima, J.M. Dawson, Phys. Rev. Lett. 43 (1979) 267.

[2] E. Esarey, P. Sprangle, J. Krall, A. Ting, IEEE Trans. Plasma Sci. 24 (1996) 252.

[3] E. Esarey, P. Sprangle, J. Krall, A. Ting, IEEE J. Quantum Electron. 33 (1997) 1879.

[4] C.G. Durfee III, H.M. Milchberg, Phys. Rev. Lett. 71 (1993) 2409.

[5] T.R. Clark, H.M. Milchberg, Phys. Rev. Lett. 78 (1997) 277.

[6] P.J. Mardahl, H.J. Lee, G. Penn, J.S. Wurtele, N.J. Fisch, Phys. Lett. A 296 (2002) 109.

[7] J.P. Verboncoeur, A.B. Langdon, N.T. Gladd, Comput. Phys. Commun. 87 (1995) 199. 